\title{
Laser-excited 580nm AlGaInP nanomembrane for visible light communications
}

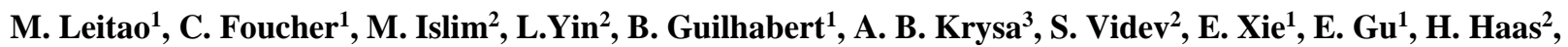 \\ N. Laurand ${ }^{1}$ and M.D. Dawson ${ }^{1}$ \\ ${ }^{1}$ Institute of Photonics, Department of Physics, SUPA, University of Strathclyde, 99 George St, Glasgow G1 1RD, UK \\ ${ }^{2}$ Li-Fi R\&D Centre, Institute for Digital Communications, University of Edinburgh, Edinburgh, EH9 3JG, UK \\ ${ }^{3}$ Electronic \& Electrical Engineering, University of Sheffield, Sheffield, UK \\ Author's e-mail address: miguel.leitao@strath.ac.uk
}

\begin{abstract}
A blue-to-yellow (580nm) AlGaInP multi-quantum-well nanomembrane colorconverter for use in GaN-based laser diode visible light communication (VLC) is presented. Preliminary tests using OFDM demonstrate free-space data transmission rates of $180 \mathrm{Mb} / \mathrm{s}$. OCIS codes: (230.0250) Optoelectronics; (250.5590) Quantum-well, -wire and -dot devices; (060.4510) Optical communications.
\end{abstract}

\section{Introduction}

The work by Akasaki, Amano [1,2] and Nakamura [3] has enabled the development of GaN-based Light Emitting Diodes (LEDs) and their subsequent widespread use in solid-state lighting. For the latter, white LEDs are usually made with a blue LED chip pumping a yellow phosphor - the blue light is partially absorbed by the phosphor and down-converted to yellow light that mixes with the non-absorbed blue, producing white. Recently, there has been great interest in extending the concept to laser diode (LD) pumped phosphors as a way to address the issue of efficiency droop that impact LEDs but also to operate in higher intensity regimes [4]. An example is the laserbased headlights that can be found in some models of cars [5,6], with laser-driven phosphors also being used in some display technologies [7].

Beyond illumination and displays, additional source functionalities are enabling other applications such as visible light communications or VLC (combining illumination with data), which takes advantage of the relatively high modulation speed of GaN LEDs and LDs. Phosphor-less LDs [8] and single-color, blue microsized LEDs ( $\mu$ LEDs) [9] have demonstrated VLC error-free data rates of $4 \mathrm{~Gb} / \mathrm{s}$ and $5 \mathrm{~Gb} / \mathrm{s}$, respectively. However, phosphor-driven sources are severely limited by the low modulation bandwidth of conventional persistent phosphors and faster modulated color converted sources are needed. An epitaxial quantum-well structure specifically designed for optical pumping with a $\mathrm{GaN}$ source is an attractive color converter candidate, as reported with II-VI [10] and III-V [11] material systems (emission at 545, 581 and $626 \mathrm{~nm}$, and $535 \mathrm{~nm}$, respectively) although these reports did not consider VLC at the time. More recently, our group has shown the integration of thin, 520nm-emitting $\mathrm{ZnCdSe}$ and 650nm-emitting AlGaInP membranes with optics and with $\mu$ LEDs and studied their VLC performance $[12,13]$.

Here, we further our work by demonstrating an AlGaInP multi-quantum-well (MQW) converting membrane that emits in the more challenging yellow spectral window. The thin-membrane format enables van der Waals bonding via liquid capillary action for integration, while the typical shorter photoluminescence lifetime and the narrower emission spectrum of MQWs compared to traditional phosphors offer benefits for VLC. In the following, we describe the design and fabrication of the structures, their characterization and report an initial demonstration of free-space VLC at $580 \mathrm{~nm}$ with a laser-driven membrane.

\section{Experimental methods}

\section{a. Design and Fabrication}

Two AlGaInP epistructures were designed and grown for a nominal emission wavelength at $580 \mathrm{~nm}$. The active region of the first structure (designated as 'anti-symmetric' henceforth) is made of $22 \operatorname{In}_{0.49} \mathrm{Ga}_{0.51} \mathrm{P}$ QWs with $\mathrm{Al}_{0.51} \mathrm{In}_{0.49} \mathrm{P}$ barriers. The QWs are unevenly distributed throughout the semiconductor's $5-\lambda$ thick active region. The second epitaxial structure is a superlattice of 70 otherwise identical QWs. Both structures have the active region enclosed between $10 \mathrm{~nm}$-thick $\operatorname{In}_{0.43} \mathrm{Ga}_{0.57} \mathrm{P}$ layers while their total thicknesses are chosen for $450 \mathrm{~nm}$ wavelength light absorption above 98\%. Growth was performed by Organometallic Vapor Phase Epitaxy (OMVPE) on a GaAs buffer layer on top of a GaAs wafer. An additional AlAs layer on the buffer side acts as etch stop. To obtain sub-micron-thick color converting membranes, the substrate is removed by wet etching following a similar process to the one in [13]; however here the sides of the membrane were also protected by an acid resistant wax to avoid lateral etching. The samples were bonded onto a $\lambda / 10$ smooth glass piece for handling and testing (Figure 1).

\section{b. Characterization and VLC demonstration}

The forward color conversion efficiency ('cc efficiency', calculated from the light emitted in the forward direction and collected by the setup) and the $450 \mathrm{~nm}$ blue light absorption of the samples ('blue abs') were measured using 
the setup depicted in Figure 1. The first set of lenses images the pump light on the sample, while the second collects the converted light (and non-absorbed pump light) and images it onto an optical power meter or a fiber-coupled spectrometer. All the lenses are similar, with a $45 \mathrm{~mm}$ focal length and a 0.612 numerical aperture and are antireflection coated for 350-700 $\mathrm{nm}$. A GaN $\mu$ LED $\left(100 \times 100 \mu \mathrm{m}^{2}\right.$ emitting area) was used as the pump light for these tests, which were performed under $\mathrm{CW}$ pumping at room temperature with no heat sinking. The yellow optical power as a function of blue optical excitation power can be seen in Figure 2. The photoluminescence (PL) spectrum was also acquired using the same setup and can be seen in Figure 2 (b). For the VLC demonstration, the LED was replaced by a 450nm LD. The set-up was otherwise similar to that in Figure 1.

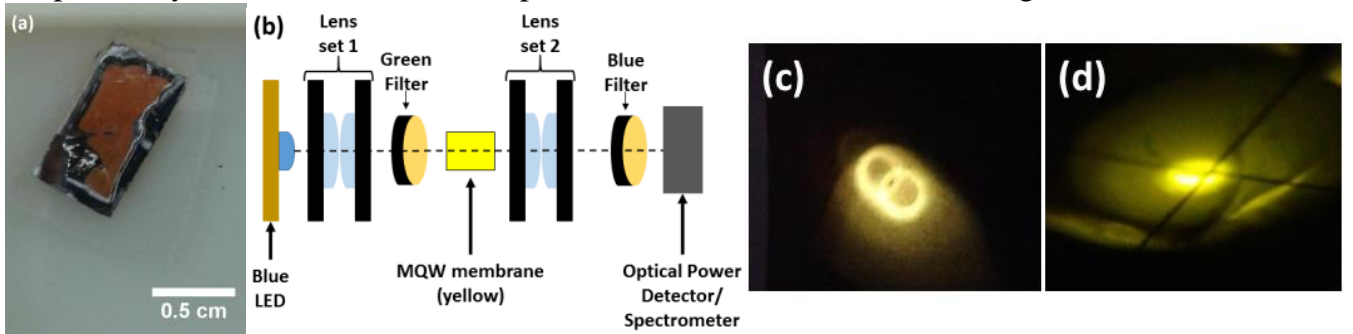

Figure 1 - (a): Picture of a sample after substrate removal. (b): Setup used for the CC efficiency, blue absorption and absorbance measurements. Yellow emission of the (c) superlattice and (d) anti-symmetric design.

\section{Results and discussions}

The cc efficiency for the superlattice and anti-symmetric design is $0.025 \%$ and $0.015 \%$, respectively, while the blue $a b s$ is around $99 \%$ and $94-95 \%$, respectively. The anti-symmetric sample's blue abs value is slightly lower than expected. Compared to a similar structure with red emission [13], the efficiency of the yellow membranes is one order of magnitude lower. This is to be expected, since these membranes emit at a wavelength closer to the direct/indirect bandgap transition of the AlGaInP alloy, and the carriers are less confined (electron confinement is $\sim 90 \mathrm{meV}$ for yellow QWs and $\sim 200 \mathrm{meV}$ for the red QWs in [13]). Furthermore, the wet etch process appears non-uniform, possibly over etching the active region, which is detrimental to the efficiency. This is especially likely to be the case for the anti-symmetric sample that has a lower blue abs. Furthermore, waveguided light in the membrane and in the glass substrate (significant in such structures) is not collected at all by the system here. The efficiency could be easily increased by using light extraction features (e.g. an integrated lens) and/or a backreflector [14]. Nevertheless, reaching efficiencies above $1 \%$ would require optimization of the growth structures as well as heat sinking.
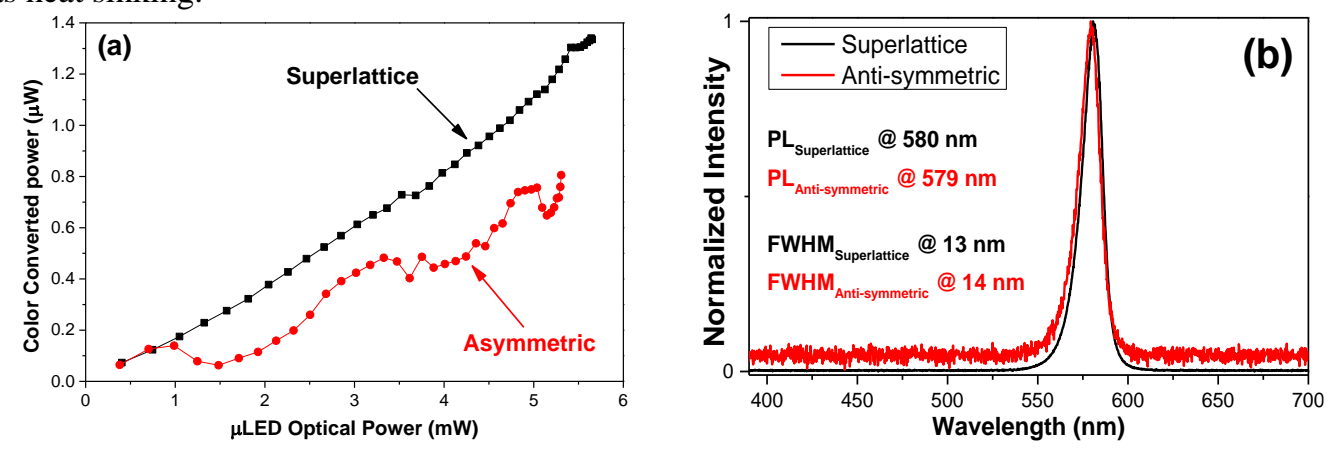

Figure 2 (a) - Yellow optical power as a function of blue optical power; (b): spectral emission of the two designs.

The spectra of both samples peaks around $580 \mathrm{~nm}$ with a full width at half maximum linewidth (FWHM) of $14 \mathrm{~nm}$. Color converters with such narrow emission are desirable for possible wavelength multiplexing VLC and for achieving a wide color gamut.

Time Correlated Single Photon Counting (TCSPC) measurements were done to assess the samples' PL lifetimes. A 450nm pulsed laser diode provided the excitation, and the PL intensity temporal decay was fitted with two exponentials. The resulting average lifetimes [14] and corresponding bandwidths [15] are $4.6 \mathrm{~ns}$ and $60 \mathrm{MHz}$ for both the superlattice and the anti-symmetric design. These results serve merely as an indication for the lower limit of bandwidth, since no information on the power density is available at this stage.

Free-space VLC demonstrations were done using a PL450B blue laser diode (TO38 ICut package) as the optical pump in an otherwise identical set-up to Figure 1. The LD was fed by a DC generator and an AC signal was transmitted from an Arbitrary Waveform Generator (AWG); an Avalanche Photodiode (APD) was used for signal detection. Direct Current Optical Orthogonal Frequency Division Multiplexing (DCO-OFDM) was used to encode the data. The results obtained for data transmission over a $10 \mathrm{~cm}$ distance (color converter to APD) can be seen in Figure 3, for both samples, plotting the Bit Error Rate (BER; ratio of received bits that have been altered 
due to noise, interference, distortion or bit synchronization errors) versus data rate. BER values were considered up to $3.8 \mathrm{E}-3$ where most forward error correction codes can operate.

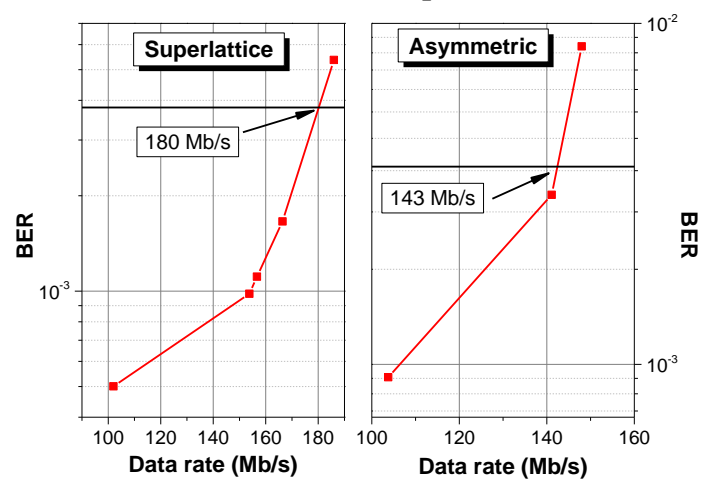

Figure 3 - BER vs data rates for the nanomembranes of the two designs

Owing to its higher $c c$ efficiency, the highest data rate, $180 \mathrm{Mb} / \mathrm{s}$, was obtained for the superlattice design at $(37 \mathrm{Mb} / \mathrm{s}$ above the anti-symmetric design). These values are below the previously reported values of $1.2 \mathrm{~Gb} / \mathrm{s}$ for the 650nm membranes [13] and are currently limited by the lower $c c$ efficiency. However, they are still at least an order of magnitude above those of conventional phosphors. Consideration on how to improve the data rates are the same as those discussed above to improve efficiency.

\section{Summary}

In conclusion, we reported - as a proof of principle - VLC with LED-driven yellow AlGaInP membranes using two different multi-quantum well structures. The yellow emission from the superlattice design achieved the highest data rate at $180 \mathrm{Mb} / \mathrm{s}$, using an OFDM modulation scheme. These results can be improved using extraction features on the nanomembrane, such as a lens. A backreflector that transmits the blue pump light and reflects the yellow converted light would also improve performance.

\section{Bibliography}

1. H. A. and M. K. and K. H. and I. Akasaki, "P-Type Conduction in Mg-Doped GaN Treated with Low-Energy Electron Beam Irradiation (LEEBI)," Jpn. J. Appl. Phys. 28, L2112 (1989).

2. I. Akasaki, H. Amano, M. Kito, and K. Hiramatsu, "Photoluminescence of Mg-doped p-type GaN and electroluminescence of GaN p-n junction LED," J. Lumin. 48-49, Par, 666-670 (1991).

3. S. Nakamura, T. Mukai, and M. Senoh, "Candela-class high-brightness InGaN/AlGaN double-heterostructure blue- light-emitting diodes," Appl. Phys. Lett. 64, (1994).

4. A. F. George, S. Al-waisawy, J. T. Wright, W. M. Jadwisienczak, and F. Rahman, "Laser-driven phosphor-converted white light source for solid-state illumination," Appl. Opt. 55, 1899-1905 (2016).

5. S. Evans, "BMW shows us how its laserheadlights and dynamic lightspot work," http://wot.motortrend.com/bmw-shows-us-how-itslaserheadlights-.

6. Z. Gale, "Audi debuts laser taillights, in-car LTE connectivity, 3D displays at 2013 CES," http://wot.motortrend.com/audi-debuts-lasertaillights-in-carlte-.

7. S. P. DenBaars, D. Feezell, K. Kelchner, S. Pimputkar, C.-C. Pan, C.-C. Yen, S. Tanaka, Y. Zhao, N. Pfaff, R. Farrell, M. Iza, S. Keller, U. Mishra, J. S. Speck, and S. Nakamura, "Development of gallium-nitride-based light-emitting diodes (LEDs) and laser diodes for energyefficient lighting and displays," Acta Mater. 61, 945-951 (2013).

8. C. Lee, C. Zhang, M. Cantore, R. M. Farrell, S. H. Oh, T. Margalith, J. S. Speck, S. Nakamura, J. E. Bowers, and S. P. DenBaars, "4 Gbps direct modulation of $450 \mathrm{~nm}$ GaN laser for high-speed visible light communication," Opt. Express 23, 16232-16237 (2015).

9. R. Ferreira, E. Xie, J. McKendry, S. Rajbhandari, H. Chun, G. Faulkner, S. Watson, A. Kelly, E. Gu, R. Penty, I. White, D. C. O’Brien, and M. Dawson, "High bandwidth GaN-based micro-LEDs for multi-Gbps visible light communications," IEEE Photonics Technol. Lett. PP, 1 (2016).

10. M. A. Haase, J. Xie, T. A. Ballen, J. Zhang, B. Hao, Z. H. Yang, T. J. Miller, X. Sun, T. L. Smith, and C. A. Leatherdale, "II-VI semiconductor color converters for efficient green, yellow, and red light emitting diodes," Appl. Phys. Lett. 96, (2010).

11. D. Schiavon, M. Binder, A. Loeffler, and M. Peter, "Optically pumped GaInN/GaN multiple quantum wells for the realization of efficient green light-emitting devices," Appl. Phys. Lett. 102, (2013).

12. J. M. M. Santos, B. E. Jones, P. J. Schlosser, S. Watson, J. Herrnsdorf, B. Guilhabert, J. J. D. McKendry, J. De Jesus, T. a Garcia, M. C. Tamargo, A. E. Kelly, J. E. Hastie, N. Laurand, and M. D. Dawson, "Hybrid GaN LED with capillary-bonded II-VI MQW color-converting membrane for visible light communications," Semicond. Sci. Technol. 30, 035012 (2015).

13. J. M. M. Santos, S. Rajbhandari, D. Tsonev, H. Chun, B. Guilhabert, A. B. Krysa, A. E. Kelly, H. Haas, D. C. O’Brien, N. Laurand, and M. D. Dawson, "Visible light communication using InGaN optical sources with AlInGaP nanomembrane down-converters," Opt. Express 24, 10020 (2016).

14. J. R. Lakowicz, Principles of Fluorescence Spectroscopy Principles of Fluorescence Spectroscopy (2006).

15. J. M. Senior and M. Y. Jamro, Optical Fiber Communications: Principles and Practice (2009).

Acknowledgements: This work was supported by the UK Engineering and Physical Sciences Research Council (EPSRC) under grant EP/K00042X/1, Ultra-parallel visible light communications (UP-VLC). 